\title{
A finite strain FE-Implementation of the Fleck-Willis gradient theory: Rate-independent versus visco-plastic formulation
}

Nielsen, K. L.; Niordson, C. F.

Published in:

European Journal of Mechanics A - Solids

Link to article, DOI:

10.1016/j.euromechsol.2019.02.010

Publication date:

2019

Document Version

Peer reviewed version

Link back to DTU Orbit

Citation (APA):

Nielsen, K. L., \& Niordson, C. F. (2019). A finite strain FE-Implementation of the Fleck-Willis gradient theory:

Rate-independent versus visco-plastic formulation. European Journal of Mechanics A - Solids, 75, 389-398. https://doi.org/10.1016/j.euromechsol.2019.02.010

\section{General rights}

Copyright and moral rights for the publications made accessible in the public portal are retained by the authors and/or other copyright owners and it is a condition of accessing publications that users recognise and abide by the legal requirements associated with these rights.

- Users may download and print one copy of any publication from the public portal for the purpose of private study or research.

- You may not further distribute the material or use it for any profit-making activity or commercial gain

- You may freely distribute the URL identifying the publication in the public portal 


\title{
A Finite Strain FE-Implementation of the Fleck-Willis Gradient Theory: Rate-independent versus Visco-Plastic formulation
}

\author{
K.L. Nielsen, C.F. Niordson \\ Department of Mechanical Engineering, Section of Solid Mechanics, Technical University of \\ Denmark, DK-2800 Kgs. Lyngby, Denmark
}

\begin{abstract}
This paper presents a numerical basis for finite strain analysis using the higher order flow theory by Fleck and Willis [2009, A mathematical basis for strain-gradient plasticity theory - part II: tensorial plastic multiplier, J. Mech. Phys. Solids, 57, 10451057]. The rate-dependent (visco-plastic) formulation shares similarities with its rateindependent (generalized J2-flow) counterpart, and a combined presentation is laid out. The visco-plastic formulation [2018, A homogenized model for size-effects in porous metals, J. Mech. Phys. Solids, DOI: 10.1016/j.jmps.2018.09.004] is revisited to set the scene for the development of the rate-independent model, but the two formulations differ substantially in the numerical implementation as the rate-independent framework possesses a challenge in determining the yield of individual plastic zones. The developed framework readily accounts for repeated loading/unloading and handles the interaction of multiple plastic zones. The method presented exploits the image analysis techniques that was first combined with the Fleck-Willis theory in Nielsen and Niordson [2014, A numerical basis for strain-gradient plasticity theory: rate-independent and rate-dependent formulations, J. Mech. Phys. Solids 63, 113-127], but extends it to finite strains by adopting an updated Lagrangian framework. Besides somewhat standard extensions to deal with finite deformations, the framework largely resembles that of small strains. In fact, the adopted image analysis can be conducted in the undeformed configuration without any approximations as it relates only to the connectivity of plastic zones on the finite element mesh. The new framework is focused on the
\end{abstract}

\footnotetext{
This article was presented at the IUTAM Symposium on Size-Effects in Microstructure and Damage Evolution at Technical University of Denmark, 2018

${ }^{*}$ Tel: +45 4525-4258, Fax: +45 4593-1475

URL: kin@mek.dtu.dk (K.L. Nielsen)
} 
deformation and localization in both uniform and notched round bars by formulating the numerical model in an axi-symmetric setup. Comparisons between the visco-plastic and the rate-independent formulation are presented.

Keywords: Finite strain analysis, Finite element method, Higher order theory

\section{Introduction}

The need to apply the family of strain gradient plasticity theories developed through the work of Gudmundson (2004); Gurtin and Anand (2005); Fleck and Willis (2009a,b) to real-life engineering applications have led to the development of specialized finite element based procedures. Fredriksson and Gudmundson (2005), and later Niordson and Legarth (2010), targeted the early visco-plastic gradient theory by Gudmundson (2004) and created a finite element based solution procedure where the displacement increments and the plastic strain rate increments are solved for in a staggered approach. The algorithms are initiated by a known stress field (for example an initial small elastic increment) based on which the increment of the plastic strain rate can be determined. A subsequent update of the plastic strain rate then allows the displacement rate to be calculated and a new stress field can be determined. These early models were employed in various investigations of size-dependent strength of e.g. biaxially stretched thin-film structures and cyclic shearing of a thin slab of material subject to micro-hard boundaries at top and bottom. The studies are, however, conducted in a rate-dependent set-up.

The strain gradient plasticity theory of Gudmundson (2004) was reformulated by Fleck and Willis (2009a,b), who put forward two Minimum Principles derived from the virtual work principle, but rather than limiting attention to visco-plasticity (ratedependent formulation) they laid out a practically applicable variational structure of the Gudmundson theory in a rate-independent formulation. This generalized flow theory of Fleck and Willis $(2009 a, b)$ is constructed such that it constitutes the limit of the corresponding rate-dependent formulation in the limit of vanishing rate-sensitivity and further reduces to the conventional J2 flow plasticity theory for vanishing material length parameters. While the rate-dependent formulation has proven to lend itself nicely to numerical implementation (Nielsen and Niordson, 2012; Dahlberg and 
Faleskog, 2012; Danas et al., 2012; Martínez-Pañeda et al., 2019), the corresponding rate-independent formulation has proven challenging. Niordson and Hutchinson (2011) made a first attempt to built the rate-independent generalized flow theory into a $2 \mathrm{D}$ plane strain finite element model and limited attention to the scalar version (see Fleck and Willis, 2009a). By adopting a Ramberg-Osgood curve for the uni-axial stress-strain relation, Niordson and Hutchinson (2011) circumvented the problem of initial yielding and, moreover, avoided elastic unloading by limiting attention to stretching of a block of material that was assumed to deform plastically throughout. The model developed in Niordson and Hutchinson (2011) yielded the first insight into what type of behavior one can expect from the rate-independent formulation, but it was highly specialized and not capable of modelling general loading conditions featuring repeated elastic-plastic loading/unloading. It was only through the work in Nielsen and Niordson $(2013,2014)$ that a rigorous finite element based solution procedure for the generalized flow theory was put forward with focus on the tensorial version of Fleck and Willis (2009b). This new procedure was largely inspired by Niordson and Hutchinson (2011) in terms of discretization and their approach to link the Minimum Principles I and II, but was also inspired by the discussion on the yield criterion in Danas et al. (2012).

The finite element models developed in Nielsen and Niordson $(2013,2014)$ largely rely upon the corresponding visco-plastic models, but they are combined with classical image analysis techniques to identify potentially active plastic zone to be enriched by a plastic multiplier and subsequently accounted for in the calculation of the incremental displacement field. Nielsen and Niordson (2014) analyzed the 1D problem of pure shear of an infinite slab, constrained between rigid platens. Hereafter, this initial model was evolved to 2D plane strain in Nielsen and Niordson (2013), where raster imagining was employed to unstructured finite element meshes, facilitating subsequent image analysis of potentially active plastic zones. The solution procedure developed in Nielsen and Niordson $(2013,2014)$ readily handles repeated elastic-plastic loading/unloading aswell as the interaction of multiple plastic zones. The model set-up is, however, limited to a small strain formulation which restricts its use in many engineering applications.

The present paper aims to develop the basis for rate-independent 2D modelling framework suitable for finite strain analysis. The goal is to lay out the theoretical 
considerations and to formulate a framework that readily can be built into the models that already exist for corresponding small strain analyses. Three finite strain benchmark tests are considered and the predictions of the rate-independent model response is compared to that of the corresponding visco-plastic (rate-dependent) formulation recently presented in Niordson and Tvergaard (2018). Following the same path taken in developing the new modelling basis, the paper is structured as follows. The rateindependent version of the Fleck and Willis theory (tensorial version) is first laid out in the context of finite strains in Section 2. The focus is on the treatment of the weak form of the equilibrium equations through a finite element solution procedure that decouples when solving for the displacement field increment and the plastic strain rate trial fields. Section 3 outlines the finite element implementation, while the model verification is laid out in Section 4 by considering three separate benchmark tests. The predictions by the developed rate-independent model is presented together with corresponding predictions by a visco-plastic (rate-dependent) model, with suppressed rate-dependency. Concluding remarks are given in Section 5.

\section{Fleck-Willis theory at Finite Strains: rate-independent and visco-plastic versions}

The model development builds upon the finite strain formulation of the Fleck and Hutchinson (2001) theory presented in Niordson and Redanz (2004) with the aim to create a corresponding framework for the Fleck and Willis (2009b) strain gradient plasticity theory. The framework adopts an updated Lagrangian formulation and uses the Jaumann rate of the Kirchhoff stress as objective measure for the elastic constitutive relation. The work follows the strategy of the updated Lagrangian conventional J2-flow plasticity as presented by McMeeking and Rice (1975) and Yamada and Sasaki (1995), and the gradient enhanced model response reduces to that of a conventional model in the limit of zero length parameter.

The total displacement vector for a given material point is charaterized by $\mathbf{u}=\mathbf{X}-$

$\mathbf{x}$, with $\mathbf{X}$ and $\mathbf{x}$ denoting positions in the current (time " $t+\Delta t$ ") and reference (time " $t$ ") configurations, respectively. The deformation gradient is given by $\mathbf{F}=\partial \mathbf{X} / \partial \mathbf{x}$, and the velocity gradient is defined by $\dot{\mathbf{e}}=\partial \dot{\mathbf{X}} / \partial \mathbf{X}$, or equivalently by $\dot{e}_{i j}=\dot{u}_{i, j}$, with 
the comma signifying derivatives in the deformed state. The anti-symmetric part of the velocity gradient, being the spin rate, is given by

$$
\dot{\omega}_{i j}=\frac{1}{2}\left(\dot{e}_{i j}-\dot{e}_{j i}\right)=\frac{1}{2}\left(\dot{u}_{i, j}-\dot{u}_{j, i}\right)
$$

while the symmetric part is the total strain rate

$$
\dot{\varepsilon}_{i j}=\frac{1}{2}\left(\dot{e}_{i j}+\dot{e}_{j i}\right)=\frac{1}{2}\left(\dot{u}_{i, j}+\dot{u}_{j, i}\right)
$$

The total strain rate is decomposed into an elastic part, $\dot{\varepsilon}_{i j}^{E}$, and a plastic part, $\dot{\varepsilon}_{i j}^{P}$, so that $\dot{\varepsilon}_{i j}=\dot{\varepsilon}_{i j}^{E}+\dot{\varepsilon}_{i j}^{P}$.

The assumption that the gradient of the plastic strain acts to store energy (governed by an energetic length parameter) as well as to dissipate energy through its rate (governed by a dissipative length parameter) is adopted in the theory of Fleck and Willis (2009b). However, the present work only considers the dissipative nature of plastic strain and the plastic strain gradients and, thus, the micro-stress, $q_{i j}=q_{i j}^{D}$, and the higher order stress, $\tau_{i j k}=\tau_{i j k}^{D}$, which are work-conjugate stress quantities to the plastic strain rate and its gradient, respectively, are assumed purely dissipative (denoted by superscript $D$ ). For both the visco-plastic and the rate-independent formulation, the dissipative quantities in the deformed configuration take the form (Gudmundson, 2004; Fleck and Willis, 2009b)

$$
q_{i j}^{D}=\frac{2}{3} \frac{\sigma_{C}}{\dot{E}^{p}} \dot{\varepsilon}_{i j}^{p}, \quad \text { and } \quad \tau_{i j k}^{D}=\frac{\sigma_{C}}{\dot{E}^{p}}\left(L_{D}\right)^{2} \dot{\varepsilon}_{i j, k}^{p}
$$

where $\sigma_{C}$ and $\dot{E}^{p}$ are identified as the effective gradient enhanced von Mises stress and the associated effective strain rate, respectively, and are given by

$$
\sigma_{C}=\sqrt{\frac{3}{2} q_{i j}^{D} q_{i j}^{D}+\left(L_{D}\right)^{-2} \tau_{i j k}^{D} \tau_{i j k}^{D}}, \quad \text { and } \quad \dot{E}^{p}=\sqrt{\frac{2}{3} \dot{\varepsilon}_{i j}^{p} \dot{\varepsilon}_{i j}^{p}+\left(L_{D}\right)^{2} \dot{\varepsilon}_{i j, k}^{p} \dot{\varepsilon}_{i j, k}^{p}} .
$$

The plastic strain gradients are denoted by $\dot{\varepsilon}_{i j, k}^{p}$, and the dissipative length parameter, $L_{D}$, scales the dissipation contribution from geometrically necessary dislocations tied to the plastic strain gradients.

\subsection{Equilibrium equations and Virtual Work Principle}

In the current (unknown) deformation configuration, the generalized virtual work principle reads (Fleck and Willis, 2009b)

$$
\int_{V}\left(\sigma_{i j} \delta \dot{\varepsilon}_{i j}+\left(q_{i j}-s_{i j}\right) \delta \dot{\varepsilon}_{i j}^{p}+\tau_{i j k} \delta \dot{\varepsilon}_{i j, k}^{p}\right) \mathrm{d} V=\int_{S}\left(T_{i} \delta \dot{u}_{i}+t_{i j} \delta \dot{\varepsilon}_{i j}^{p}\right) \mathrm{d} S
$$


and from this the governing equilibrium equations (the strong form) can be identified as

$$
\begin{aligned}
& q_{i j}-\tau_{i j k, k}-s_{i j}=0 \quad\text { (Higher order equilibrium }) \\
&\left.\sigma_{i j, j}=0 \quad \text { (Conventional equilibrium }\right) .
\end{aligned}
$$

Here, $\sigma_{i j}$ is the symmetric Cauchy stress tensor and $s_{i j}=\sigma_{i j}-\delta_{i j} \sigma_{k k} / 3$ its deviatoric part, with $\delta_{i j}$ denoting Kronecker's delta. The right-hand side of Eq. (5) includes both conventional tractions, $T_{i}=\sigma_{i j} n_{j}$, and higher order terms, $t_{i j}=\tau_{i j k} n_{k}$, with $n_{k}$ being the outward unit normal to the surface, $S$, that bounds the volume, $V$, in the current configuration.

Fleck and Willis (2009b) put forward two Minimum Principles I and II, based on the equilibrium equations, Eqs. (6)-(7), which serve as the backbone in existing numerical implementations and solution procedures under the assumption of small strains (see e.g. Danas et al., 2012; Nielsen and Niordson, 2013, 2014). The present framework largely lean on these existing contributions by adopting Minimum Principle I, holding the constitutive relations for the gradient related quantities, to deliver the plastic strain rate field (except for unknown plastic multipliers for the individual active plastic zones with homogeneous boundary conditions in the rate-independent formulation). However, Minimum principle II is modified based on an updated Lagrangian finite strain approach to solve for displacement increments based on the virtual work principle in Eq. (5). Thus, the numerical solution procedure relies on a 2-step approach as follows; In Step 1, the plastic strain rate (trial) field is first calculated based on a known stress field in the updated deformed configuration (based on Minimum Principle I). In the rate-independent formulation, when the domain is subject to homogeneous boundary conditions, this yields the plastic field apart from a number of multipliers each specific to the individual potentially active plastic zones. In Step 2, the incremental displacement field is subsequently determined from the generalized virtual work principle in a forward Euler time stepping procedure and it is through this second step that the plastic multipliers are evaluated ${ }^{1}$.

\footnotetext{
${ }^{1}$ Note that the plastic multipliers are not introduced in the visco-plastic setting, nor are they introduced for a plastic zone subject to inhomogeneous boundary conditions in the time-independent
} 
The Minimum Principle I is responsible for the gradient related constitutive behavior and, throughout, it is considered in an updated deformed configuration. Omitting the energetic higher order stress contribution, this reads for the rate-independent version;

$$
H=\inf _{\dot{\varepsilon}_{i j}^{p *}} \int_{V}\left(\sigma_{F}\left[E^{p}\right] \dot{E}^{p *}-s_{i j} \dot{\varepsilon}_{i j}^{p *}\right) \mathrm{d} V-\int_{S} t_{i j} \dot{\varepsilon}_{i j}^{p *} \mathrm{~d} S
$$

and for the visco-plastic (rate-dependent) version;

$$
H=\inf _{\dot{\varepsilon}_{i j}^{p *}} \int_{V}\left(\Phi\left[E^{p}, \dot{E}^{p}\right]-s_{i j} \dot{\varepsilon}_{i j}^{p *}\right) \mathrm{d} V-\int_{S} t_{i j} \dot{\varepsilon}_{i j}^{p *} \mathrm{~d} S
$$

Here, $\sigma_{F}\left[E^{p}\right]$ denotes the current pointwise flow stress at the deformation level $E^{p}$, and $\Phi\left[E^{p}, \dot{E}^{p}\right]$ denotes the visco-plastic potential. For each increment, both the displacement field $u_{i}$ and the stress field, $\sigma_{i j}$, are known and based on an updated configuration. Minimum Principle I delivers a plastic strain rate (trial) field. Stationarity of the functionals in Eqs. (8)-(9) results in

$$
\int_{V}\left(q_{i j}^{D} \delta \hat{\varepsilon}_{i j}^{p}+\tau_{i j k}^{D} \delta \hat{\varepsilon}_{i j, k}^{p}\right) \mathrm{d} V=\int_{V} s_{i j} \delta \hat{\varepsilon}_{i j}^{p} \mathrm{~d} V+\int_{S} t_{i j} \delta \hat{\varepsilon}_{i j}^{p} \mathrm{~d} S
$$

where $\hat{\varepsilon}_{i j}^{p}$ denotes the plastic strain rate field associated with either the generalized flow theory (rate-independent, $\dot{\varepsilon}_{i j}^{p}=\lambda \hat{\varepsilon}_{i j}^{p}$ ) or the visco-plastic formulation (rate-dependent, $\dot{\varepsilon}_{i j}^{p}=\hat{\varepsilon}_{i j}^{p}$ ). The finite element discretization of Eq. (10) is presented in Section 3.

Minimum Principle II is based on the Principle of Virtual Work in Eq. (5). In the visco-plastic formulation, the Minimum Principle I has directly delivered the plastic strain rate field such that $\delta \dot{\varepsilon}_{i j}^{p}=\delta \hat{\varepsilon}_{i j}^{p}$ and $\delta \dot{\varepsilon}_{i j, k}^{p}=\delta \hat{\varepsilon}_{i j, k}^{p}$, so that the Principle of Virtual Work in the deformed configuration takes the form

$$
\begin{array}{r}
\int_{V} \sigma_{i j} \delta \dot{\varepsilon}_{i j} \mathrm{~d} V+\overbrace{\int_{V}\left(q_{i j}^{D} \delta \dot{\varepsilon}_{i j}^{p}+\tau_{i j k}^{D} \delta \dot{\varepsilon}_{i j, k}^{p}\right) \mathrm{d} V-\int_{V} s_{i j} \delta \dot{\varepsilon}_{i j}^{p} \mathrm{~d} V-\int_{S} t_{i j} \delta \dot{\varepsilon}_{i j}^{p} \mathrm{~d} S}^{\text {vanishes due to Eq. (10) }} \\
=\int_{S} T_{i} \delta \dot{u}_{i} \mathrm{~d} S
\end{array}
$$

Part of the left hand side vanishes due to the stationarity requirement, Eq. (10), and the conventional Principle of Virtual Work is obtained;

$$
\int_{V} \sigma_{i j} \delta \dot{\varepsilon}_{i j} \mathrm{~d} V=\int_{S} T_{i} \delta \dot{u}_{i} \mathrm{~d} S
$$

model. 
In contrast, the variation in the plastic strain increment and the associated variation in gradients in the rate-independent formulation read; $\delta \dot{\varepsilon}_{i j}^{p}=\delta \lambda \hat{\varepsilon}_{i j}^{p}+\lambda \delta \hat{\varepsilon}_{i j}^{p}$ and $\delta \dot{\varepsilon}_{i j, k}^{p}=$ $\delta \lambda \hat{\varepsilon}_{i j, k}^{p}+\lambda \delta \hat{\varepsilon}_{i j, k}^{p}$. That is, the variation of the plastic strain rate and its gradients are separated into the variation in its magnitude (through $\delta \lambda$ ) and the variation in the shape of the field (through the trial field $\delta \hat{\varepsilon}_{i j}^{p}$ ). The Principle of Virtual Work in the deformed configuration thereby reads

$$
\begin{aligned}
& \int_{V}\left(\sigma_{i j} \delta \dot{\varepsilon}_{i j}-\right.s_{i j} \delta \lambda \hat{\varepsilon}_{i j}^{p}+\overbrace{\left(q_{i j}^{D} \hat{\varepsilon}_{i j}^{p}+\tau_{i j k}^{D} \hat{\varepsilon}_{i j, k}^{p}\right)}^{\sigma_{C} \hat{E}^{p}} \delta \lambda) \mathrm{d} V+ \\
&+\underbrace{\int_{V}\left(q_{i j}^{D} \delta \hat{\varepsilon}_{i j}^{p}+\tau_{i j k}^{D} \delta \hat{\varepsilon}_{i j, k}^{p}\right) \mathrm{d} V-\int_{V} s_{i j} \delta \hat{\varepsilon}_{i j}^{p} \mathrm{~d} V-\int_{S} t_{i j} \delta \hat{\varepsilon}_{i j}^{p} \mathrm{~d} S}_{\text {vanishes due to Eq. (10) }} \\
&=\int_{S}\left(T_{i} \delta \dot{u}_{i}+t_{i j} \delta \lambda \hat{\varepsilon}_{i j}^{p}\right) \mathrm{d} S
\end{aligned}
$$

Also here, part of the left hand side vanishes due to the stationarity requirement, Eq. (10), and thus Minimum Principle II takes the form

$$
\int_{V}\left(\sigma_{i j} \delta \dot{\varepsilon}_{i j}-s_{i j} \delta \lambda \hat{\varepsilon}_{i j}^{p}+\sigma_{C} \hat{E}^{p} \delta \lambda\right) \mathrm{d} V=\int_{S}\left(T_{i} \delta \dot{u}_{i}+t_{i j} \delta \lambda \hat{\varepsilon}_{i j}^{p}\right) \mathrm{d} S
$$

Note, again, that the variation in the plastic strain rate trial field, $\delta \hat{\epsilon}_{i j}^{p}$ has been eliminated in both Eqs. (12) and (14) due to Minimum Principle I, which is fulfilled separately. Hence, the equations involve only the incremental displacement field, $\delta \dot{u}_{i}$, in addition to the plastic multiplier, $\delta \lambda$, in the rate-independent case with plastic zones subject to homogeneous boundary conditions.

The current configuration referred to in Eqs. (12) and (14) is unknown and, hence, the virtual work principles are rewritten in the reference configuration on incremental form by use of the first Piola-Kirchhoff stress, $P_{i j}$, the Kirchhoff stresses, $\tau_{i j}$, the effective Kirchhoff stress, $\tau_{C}$, the nominal conventional tractions, $T_{i j}^{0}$, and nominal higher order tractions, $t_{i j}^{0}$. The incremental form of the principle of virtual work for the rate-independent formulation thereby reads ${ }^{2}$

$$
\int_{V_{0}}\left(\dot{P}_{i j} \delta \dot{u}_{i, j}-\dot{\tau}_{i j} \delta \lambda \hat{\varepsilon}_{i j}^{p}+\dot{\tau}_{C} \delta \lambda \hat{E}^{p}\right) \mathrm{d} V=\int_{S_{0}}\left(\dot{T}_{i}^{0} \delta \dot{u}_{i}+\dot{t}_{i j}^{0} \delta \lambda \hat{\varepsilon}_{i j}^{p}\right) \mathrm{d} S
$$

\footnotetext{
${ }^{2}$ The corresponding visco-plastic version is readily extracted by letting $\delta \lambda=0$ and substituting the appropriate plastic strain rate field as $\dot{\varepsilon}_{i j}^{p}=\hat{\varepsilon}_{i j}^{p}$ in the remaining.
} 
By taking as offset the updated Lagrangian finite element method put forward by McMeeking and Rice (1975) for conventional plasticity, Eq. (15) can be rewritten by introducing the Jaumann rate of the Kirchhoff stress $\left(\stackrel{\nabla}{\tau}_{i j}=\dot{\tau}_{i j}-\dot{\omega}_{i k} \tau_{k j}-\tau_{i k} \dot{\omega}_{j k}\right)$ such that

$$
\begin{aligned}
\int_{V_{0}}\left(\stackrel{\nabla}{\tau}_{i j} \delta \dot{\varepsilon}_{i j}-\sigma_{i j}\left(2 \dot{\varepsilon}_{i k} \delta \dot{\varepsilon}_{k j}-\dot{u}_{k, j} \delta \dot{u}_{k, i}\right)\right)-\stackrel{\nabla}{\tau}_{i j} \delta \lambda \hat{\varepsilon}_{i j}^{p} & +\left(\dot{J}_{\sigma_{C}}+J \dot{\sigma}_{C}\right) \delta \lambda \hat{E}^{p} \mathrm{~d} V \\
& =\int_{S_{0}}\left(\dot{T}_{i}^{0} \delta \dot{u}_{i}+\dot{t}_{i j}^{0} \delta \lambda \hat{\varepsilon}_{i j}^{p}\right) \mathrm{d} S
\end{aligned}
$$

or rewritten into a form closely associated with a stationarity requirement of Minimum

Principle II as formulated by Fleck and Willis (2009b) by introducing $\stackrel{\nabla_{i j}}{\tau_{i j}}=\mathcal{L}_{i j k l}\left(\dot{\varepsilon}_{k l}-\right.$ $\left.\lambda \hat{\varepsilon}_{k l}^{p}\right)$ and $\dot{\sigma}_{C}=h\left[E^{p}\right] \lambda \hat{E}^{p}$ such that

$$
\begin{aligned}
& \int_{V_{0}}\left(\mathcal{L}_{i j k l}\left(\dot{\varepsilon}_{i j}-\lambda \hat{\varepsilon}_{i j}^{p}\right)\left(\delta \dot{\varepsilon}_{k l}-\delta \lambda \hat{\varepsilon}_{k l}^{p}\right)-\sigma_{i j}\left(2 \dot{\varepsilon}_{i k} \delta \dot{\varepsilon}_{k j}-\dot{u}_{k, j} \delta \dot{u}_{k, i}\right)\right) \mathrm{d} V \\
& \int_{V_{0}}\left(\dot{J} \sigma_{C} \delta \lambda \hat{E}^{p}+J h\left[E^{p}\right] \lambda \delta \lambda\left(\hat{E}^{p}\right)^{2}\right) \mathrm{d} V=\int_{S_{0}}\left(\dot{T}_{i}^{0} \delta \dot{u}_{i}+\dot{t}_{i j}^{0} \delta \lambda \hat{\varepsilon}_{i j}^{p}\right) \mathrm{d} S
\end{aligned}
$$

Here, $h\left[E^{p}\right]=\mathrm{d} \sigma_{C} / \mathrm{d} E^{p}$ and $J=\operatorname{det}(\mathbf{F})$ (with $J$ being unity in an updated Lagrangian framework). It is worth noting that two additional terms have arisen in the finite strain formulation of Minimum Principle II; i) the first being the ordinary term related to the stress stiffness known from conventional finite strain plasticity, and ii) the second being a term related to the volume change rate (further discussed in Appendix A).

\section{Finite element implementation and solution procedure}

The following numerical formulation is largely inspired by the work in Nielsen and Niordson $(2013,2014)$, where a solution procedure for the Fleck and Willis (2009b) theory was developed within a small strain framework based on Minimum Principles I and II. The current finite element procedure is based on the stationarity requirement in Eq. (10) to obtain the plastic strain rate field in the visco-plastic case or the plastic strain rate trial field in the time-independent case. Subsequently, the visco-plastic theory employs Eq. (17) (with $\delta \lambda=0$ ) to deliver the incremental displacement field and the rate-independent theory employs Eq. (17) to deliver the incremental displacement field and the plastic multipliers for "potentially active" zones subject to homogeneous 
boundary conditions. A standard finite element interpolation is employed such that

$$
u_{i}=\sum_{n=1}^{N_{I I}} N_{i}^{(n)} U^{(n)} \quad \text { and } \quad \hat{\varepsilon}_{i j}^{p}=\sum_{n=1}^{N_{I}} M_{i j}^{(n)} \hat{\varepsilon}^{(n)}
$$

where quadratic shape functions, $N_{i}^{(n)}$, are used for the displacement field and linear shape functions, $M_{i j}^{(n)}$, are used for the plastic strain rate field. Here, $U^{(n)}$ and $\hat{\varepsilon}^{p(n)}$ are the nodal values of the two field quantities, while $N_{I}$ and $N_{I I}$ denotes the number of degrees of freedom for the element displacement field $\left(N_{I I}=16\right)$ and plastic strain rate field $\left(N_{I}=12\right)$, respectively. As in the small strain formulation, the new finite strain incremental finite element solution procedure progresses in two successive steps as follows.

Step 1. Based on the known stress state in an updated deformed configuration (known displacement field, $u_{i}$, and plastic strain field in terms of the effective plastic strain, $\left.E^{p}\right)$, a plastic strain rate field is determined from the stationarity requirement (see Eq. (10)) consistent with Minimum Principles I (see Eqs. (8) and (9)). A system of equations is obtained as

$$
\begin{aligned}
\int_{V_{e}} \frac{\sigma_{C}}{\hat{E}^{p}}\left(\frac{2}{3} M_{i j}^{(n)} M_{i j}^{(m)}+L_{D}^{2} M_{i j, k}^{(n)} M_{i j, k}^{(m)}\right) \mathrm{d} V_{e} \cdot \hat{\varepsilon}^{p(m)} & \\
& =\int_{V_{e}} s_{i j} M_{i j}^{(n)} \mathrm{d} V_{e}+\int_{S_{e}} t_{i j} M_{i j}^{(n)} \mathrm{d} S_{e}
\end{aligned}
$$

This nonlinear system is solved iteratively for $\hat{\varepsilon}_{i j}^{p}$ (see Niordson and Hutchinson, 2011; Nielsen and Niordson, 2013, 2014). The plastic strain rate field is directly obtained from Eq. (19) in the visco-plastic formulation, but it only delivers the plastic strain rate (trial) field to within a plastic multiplier, $\lambda$, for the rate-independent model (except for plastic zones subject to in-homogeneous boundary conditions). The trial field obtained is therefore normalized in each iteration to form a unit field, and convergence of the solution is deemed sufficiently accurate when the relative change in the solution vector and the individual components is below $10^{-3}$ and $10^{-2}$, respectively, for two successive iterations. The incremental displacement field, as well as any plastic multipliers are subsequently determined through Step 2 below.

Step 2 involves a non-standard numerical procedure to determine the incremental displacement field and any plastic multipliers associated with plastic zone in rateindependent model. The situation without plastic multipliers is straight forward and 
follows the methodology described in Niordson and Tvergaard (2018), whereas the case where plastic multipliers must be determined involves dividing the material into "potentially active" (elastic-plastic) and "inactive" (elastic response) regions, so that $V_{0}=V_{0}^{p l}+V_{0}^{e l}$. One can thereby express the variational principle, Eq. (17), as

$$
\begin{aligned}
& \sum_{k=1}^{K} \int_{V_{0}^{p l(k)}}\left(\mathcal{L}_{i j k l}\left(\dot{\varepsilon}_{i j}-\lambda^{(k)} \hat{\varepsilon}_{i j}^{p}\right)\left(\delta \dot{\varepsilon}_{k l}-\delta \lambda^{(k)} \hat{\varepsilon}_{k l}^{p}\right)+h\left[E^{p}\right] \lambda^{(k)}\left(\dot{E}^{p *}\right)^{2} \delta \lambda^{(k)}+\dot{J} \sigma_{C} \delta \lambda^{(k)} \hat{E}^{p}\right) \mathrm{d} V^{p l(k)}+ \\
& \int_{V_{0}^{e l}} \mathcal{L}_{i j k l} \dot{\varepsilon}_{i j} \delta \dot{\varepsilon}_{k l} \mathrm{~d} V^{e l}-\int_{V_{0}} \sigma_{i j}\left(2 \dot{\varepsilon}_{i k} \delta \dot{\varepsilon}_{k j}-\dot{u}_{k, j} \delta \dot{u}_{k, i}\right) \mathrm{d} V=\int_{S_{0}}\left(\dot{T}_{i}^{0} \delta \dot{u}_{i}+\dot{t}_{i j}^{0} \delta \lambda \hat{\varepsilon}_{i j}^{p}\right) \mathrm{d} S
\end{aligned}
$$

where, $K$ is the number of "potentially active" plastic zones specified as connected material regions for which; $\sqrt{2 \dot{\varepsilon}_{i j}^{p *} \dot{\varepsilon}_{i j}^{p *} / 3}$ is greater than a predefined threshold (here taken to be $10^{-3}$ ), chosen small relative to the maximum value (being unity) in the normalized trial field. The classical image analysis technique known as Connected Component Labeling is then adopted for the identification of plastic zones (see Haralick and Shapiro, 1992; Nielsen and Niordson, 2014). In fact, since the identification of the "potentially active" plastic zone is all about determining which elements are attached to a specific region of the mesh the image analysis can be conducted on the initial un-deformed mesh. For a "potentially active" zone to be classified as actively plastically deforming (see also discussion in Danas et al., 2012), the value of $H$ in Minimum Principle I integrated over "potentially active" zone has to be non-positive. Equivalently this non-local yield criterion can be expressed as

$$
\Phi_{H}^{(k)}=-\left(\int_{V^{p l(k)}}\left(\sigma_{F}\left[E^{p}\right] \hat{E}^{p}-s_{i j} \hat{\varepsilon}_{i j}^{p}\right) \mathrm{d} V^{p l(k)}-\int_{S^{p l(k)}} t_{i j} \hat{\varepsilon}_{i j}^{p} \mathrm{~d} S^{p l(k)}\right) \geq 0
$$

which is evaluated, within the individual plastic zones, for the unit magnitude field that fulfills Eq. (19).

Requiring Eq. (20) to hold for any kinematically admissible variation of the displacement field, $\delta u_{i}$, and plastic multipliers, $\delta \lambda^{(k)}$, two discretized systems of equations can be written as

$$
\begin{aligned}
& \int_{V_{0 e}} \mathcal{R}_{i j k l} B_{i j}^{(n)} B_{k l}^{(m)}+\sigma_{i j}\left(N_{k, j}^{(m)} N_{k, i}^{(n)}-2 B_{i k}^{(m)} B_{k j}^{(n)}\right) \mathrm{d} V_{e} \cdot \dot{U}^{(m)}-\int_{V_{0 e}} \mathcal{R}_{i j k l} B_{i j}^{(n)} \hat{\varepsilon}_{k l}^{p} \mathrm{~d} V_{e} \cdot \lambda^{(k)}=\int_{S_{0 e}} \dot{T}_{i}^{0} N_{i}^{(n)} \mathrm{d} S_{e} \\
& -\int_{V_{0 e}} \mathcal{R}_{i j k l} B_{i j}^{(m)} \hat{\varepsilon}_{k l}^{p}+B_{k k}^{(m)} \sigma_{C} \hat{E}^{p} \mathrm{~d} V_{e} \cdot \dot{U}^{(m)}+\int_{V_{0 e}}\left(\mathcal{R}_{i j k l} \hat{\varepsilon}_{i j}^{p} \hat{\varepsilon}_{k l}^{p}+h\left[E^{p}\right]\left(\hat{E}^{p}\right)^{2}\right) \mathrm{d} V_{e} \cdot \lambda^{(k)}=\int_{S_{0 e}} \dot{t}_{i j}^{0} \hat{\varepsilon}_{i j}^{p} \mathrm{~d} S_{e}
\end{aligned}
$$


with $B_{i j}^{(n)}=\left(N_{i, j}^{(n)}+N_{j, i}^{(n)}\right) / 2$. The two systems of equations are coupled and form a system of size; $\left[U_{\text {d.o.f }}+K\right] \times\left[U_{\text {d.o.f }}+K\right]$, with $U_{\text {d.o.f }}$ denoting the number of displacement degrees of freedom. Once a solution has been obtained from this system of equations, the requirement of positive plastic multipliers; $\lambda^{(k)} \geq 0(k=1,2, . . K)$ is addressed. Only if $\lambda^{(k)}>0$ is the zone considered "active", whereas if $\lambda^{(k)} \leq 0$ the zone is "inactive" and removed from the system of equations, which subsequently is solved again. The processes of eliminating "inactive" plastic zones is repeated until all "potentially active" plastic zone satisfy; $\Phi_{H}^{(k)}>0$ and $\lambda^{(k)}>0$, after which they are declared active, and the incremental solution is updated.

Note that the finite strain version of the Minimum Principle II in Eq. (17) reduces to that of Nielsen and Niordson $(2013,2014)$ when disregarding the stress stiffness and volume change rate, and thus the numerical implementation largely builds on the small strain models.

It is apparent from Eqs. (22)-(23) that the system matrix becomes non-symmetric when plasticity initiates as a volume change rate term enters into one of the coupling matrices. However, due to plastic incompressibility, the volume change rate is solely tied to the elastic deformation and it is hence very limited. In fact, sufficient accuracy in the model prediction has been achieved, for all problems considered in the present paper, by neglecting the volume change rate and only considering the symmetric part of the system matrix (see Appendix A for an example).

As stated earlier, the visco-plastic formulation offers a more direct solution to the displacement field, when the plastic strain rate field has been determined, and it does not involve special attention to the plastic zones. Thus, the implementation into a finite element model is standard and largely follows a conventional finite strain modelling (see Niordson and Tvergaard, 2018, for further details).

\section{Model verification: rate-independent versus visco-plastic formulation}

The finite strain, finite element based, model incorporating the rate-independent formulation of Fleck and Willis (2009b) is verified through three benchmark tests. A parameter study is conducted for each test, focusing on the finite strain aspects and the numerical challenges related to the rate-independent formulation. When considered 
of interest, the benchmark test is solved using both the rate-independent generalized flow formulation and the visco-plastic formulation. A small strain rate hardening exponent $(m=0.002)$ is imposed in the visco-plastic model enabling a close comparison of the finite strain results obtained. The material is assumed to be elastically isotropic with $E$ and $\nu$ denoting Young's modulus and Poisson's ratio, respectively. Hardening is assumed to follow a power-law relation according to $\sigma_{F}=\sigma_{Y}\left(1+E E^{p} / \sigma_{Y}\right)^{N}$, with $N$ denoting the strain hardening exponent. The flow potential for the visco-plastic analyses is taken as

$$
\Phi\left[E^{p}, \dot{E}^{p}\right]=\sigma_{F}\left[E^{p}\right] \frac{\dot{\varepsilon}_{0}}{m+1}\left(\frac{\dot{E}^{p}}{\dot{\varepsilon}_{0}}\right)^{m+1}
$$

with $m$ denoting the rate hardening exponent, and $\dot{\varepsilon}_{0}$ denoting the reference strain rate. Values of the material parameters employed are given in Table A.1.

In addition to three benchmark tests, corresponding conventional J2-flow model simulations for the notched round tensile bars have been conducted in the commercial software ANSYS. Closely matching load-deflection curves, and deformed configurations, were achieved by systematically letting the length parameter approach zero in both gradient enhanced models. This comparison is, however, omitted in the following.

\subsection{Necking of round uni-axial tensile bars}

The first benchmark test considers necking of a round uni-axial tensile bar of length, $2 H$, and radius, $W$. Due to symmetry, only the top half of the bar is modelled, such that a necking region is forced to develop at the bottom boundary $\left(x_{2}=0\right)$ by prescribing an imperfection in the radius of the form; $\delta\left(x_{2}\right)=-\delta_{0}\left(1+\cos \left(\pi x_{2} / H\right)\right) / 2$ (see Fig. 1a). Throughout, the initial aspect ratio of the domain is $H / W=3$ and a structured finite element mesh is employed (see Fig. 1b). Uni-axial stretching is prescribed at the top boundary (at $x_{2}=H$ ). The stretch is prescribed such that the far-field uni-axial initial strain rate equals the reference strain rate for the visco-plastic material model. The free (right) boundary and the top boundaries are both assumed to be micro-free $\left(\dot{\varepsilon}_{i j}^{p} \neq 0\right)$ in the higher order model, and symmetry conditions are applied at $x_{2}=0$ and along the center axis (at $\left.x_{1}=0\right)$.

Figure 2a demonstrates the ability of the models to predict diffuse Considère necking that develops shortly after the peak load is attained at $\varepsilon=N(=0.05)$. The load 
carrying capacity essentially builds up with the strain hardening, plastically deforming the entire bar uniformly, until shortly after the Considère criterion, $\partial \sigma / \partial \varepsilon=\sigma$, is reached. The plastic flow hereafter localizes in a region that remains plastic and the bar continues to thin upon further stretching, while material outside the localization region unloads elastically. A deformed configuration of an advanced neck is depicted in Fig. 2b for a small length parameter (resembling a conventional material). The left of Fig. 2b shows the gradient enhanced effective plastic strain, whereas the right of Fig. 2b shows the current active plastic zone. As expected, both the plastic straining and the active plastic zone localize as necking intensifies.

The match between the new rate-independent model and a corresponding viscoplastic (rate-dependent) model is illustrated throughout Figs. 2-3. It is predicted that an increase in the strain hardening will postpone neck development, in agreement with the Considère criterion (see Fig. 3a), whereas the effect of plastic strain gradient hardening delays the evolution of the neck when increasing the length parameter (see Fig. 2a and Fig. 3). In contrast, localization is enhanced by enlarging the prescribed imperfection in the bar (see Fig. 3b). The delayed evolution of the neck when accounting for strain gradient hardening is also illustrated in Fig. 2c, where the deformed configuration of an advanced neck is depicted when $L_{D} / W=0.2$. To enable a direct comparison, the deformed states in Fig. 2b and Fig. 2c are both extracted when the load carrying capacity has dropped to $F /\left(A_{0} \sigma_{Y}\right) \approx 0.7$. It is evident that the effect of strain gradients is to postpone necking, as well as to enlarge the active plastic zone size.

\subsection{Localization in notched round tensile bars}

The second benchmark test focuses attention on a more complex loading scenario by considering plastic flow localization in round notched tensile bars. Symmetry is also exploited here so that only the top half of the bar is modelled. The half initial length of the bar is denoted, $H$, the radius, $W$, and the notch radius, $R$. The initial geometry is defined by $H / W=3$ and $R / W=0.5$ (see Fig. 4a). A more sophisticated mesh is employed to test the adopted raster imaging technique (see Fig. 4b). At the top boundary $\left(x_{2}=H\right)$, the domain is constrained from contracting $\left(\dot{u}_{1}=0\right)$, while a prescribed elongation is enforced in the $x_{2}$-direction such that the far-field uni-axial 
initial strain rate equals the reference strain rate for the visco-plastic material. The free (right) boundary and the top boundary are both kept micro-free $\left(\dot{\varepsilon}_{i j}^{p} \neq 0\right)$, and symmetry conditions are prescribed along $x_{2}=0$ and along the center axis (at $x_{1}=0$ ).

Figure 5a depicts load-deflection curves for a spectrum of the material length parameter and for a low value of conventional strain hardening $(N=0.05)$. An increase in the load carrying capacity is predicted for larger length parameters, due to suppression of neck localization as also observed in deformation of uniform round bars (see Section 4.1). Similarly, the influence of the additional hardening owing to plastic strain gradients retards the plastic flow localization such that the notched bar thins at a slower pace when increasing the length parameter. This is also seen from Fig. 5b, showing the relative thinning of the notched cross-section $\left(\right.$ at $\left.x_{2}=0\right)$ as function of overall strain. Deformed configurations of the notched bars are depicted in Fig. 6a for a small length parameter (resembling a conventional material) and in Fig. 6b for a larger length parameter. To the left in Figs. $6 \mathrm{a}$ and $6 \mathrm{~b}$ the gradient enhanced effective plastic strain is shown, whereas the right shows the active plastic zones. As for necking in uniform bars, an increased material length parameter (enhancing gradient hardening) leads to larger active plastic zones. The effect of combining high conventional strain hardening and gradient hardening is further demonstrated in Fig. 7.

Included in Figs. 5 and 7 are the predictions from the flow theory (rate-independent model) as-well as the visco-plastic theory (rate-dependent model). A very good agreement between the two models is obtained. However, it is worth noting that the viscoplastic model for a strain rate hardening exponent as low as $m=0.002$ requires a very large number of load increments to obtain convergence in the plastic strain rate field. Similarly, small load increments must also be employed for the time-independent model as the yield condition (see Eq. (21)) tends to drift away from zero if too few increments are employed in combination with an insufficiently strict convergence criterion. The drift of the yield criterion is either seen as a sudden elastic unloading of the structure or, simply as a slow drift from the correct solution. In fact, the visible small discrepancy between some of the curves in Figs. 5 and 7 are partly assigned to this issue and partly to the finite element discretization. In fact, it was found that the model based on the generalized flow theory requires a finer spatial discretization in regions 
undergoing severe plastic deformation, compared to the visco-plastic model. This is tied to the entire element being either elastic or elastic-plastic (enriched by a plastic multiplier) in the rate-independent model, whereas the visco-plastic model allows for elastic versus elastic-plastic deformation on the Gauss point level (as in a conventional plasticity modelling framework).

\subsection{Localization in a large double notched annular ring}

The last benchmark test considers stretching and rotation of a double notched annular ring by shifting the meshed domain in Fig. 4a a distance of $R_{C} / H=10^{6}$ in the $x_{1^{-}}$ direction (radial direction). The setup approximates plane strain tension of a double notched bar as the hoop strain in the axi-symmetric model will approach zero for $R_{C} \rightarrow$ $\infty$. That is, the response of the domain stretched to a prescribed deformation in the $x_{2^{-}}$ direction must coincide with that of a corresponding domain stretched while rotated about the out-of-plane axis as the strain related to the rotation must be negligible. In the following, the domain is restricted to contract in the transverse direction at top/bottom (though micro-free, such that $t_{i j}=0$ ).

Figure 8 displays the load-deflection curves for the rate-independent model for two cases of conventional strain hardening $(N=[0.05,0.2])$, and for two distinct length parameters $\left(L_{D} / W=[0.02,0.4]\right)$. Included are the results of two distinct loading scenarios; i) the first is stretching of the notched domain along the $x_{2}$-axis (see Fig. 9a), and ii) the second is stretching of the notched domain while it is rotated about the out-of-plane axis at a rate of $\dot{\theta} /(\dot{\Delta} / H)=4 \pi / 3$ in the clockwise direction (see Fig. 9b). This second prescribed loading condition initiates as stretching along the $x_{2}$-axis, but continues along an axis that undergoes finite rotation (see Fig. 4c). Practically coinciding curves are predicted for all cases depicted in Fig. 8, confirming that the convective rates are well capable of handling the superimposed rigid body rotation (see Fig. 8).

The set-up considered in this benchmark test poses the challenge of having two active plastic zones developing, one from each notch, which merge when the plastic deformation spans the entire notch region. Figure 9 illustrates the evolution of plastic zones, with the top row showing a sequence of deformed stages when the domain is stretched along the $x_{2}$-axis, and the bottom row shows corresponding stretched stages for the case where the domain also undergoes finite rotations. It is demonstrated that 
the model readily treats the interaction of multiple plastic zone and subsequent elastic unloading above/below the large strained notch region - even in a domain undergoing finite rotations.

\section{Concluding remarks}

The finite element based solution procedure developed in Nielsen and Niordson (2013, 2014) for small strain analysis is extended to account for finite strains. The structure of the solution method is based on handling Minimum Principle I (delivering the plastic strain rate (trial) field) in an updated deformed configuration, where the Cauchy stresses are known. The incremental displacement field is determined in an updated Lagrangian framework together with multipliers from the individual plastic zones for the rate-independent (generalized flow) theory (see Section 2). The new framework is implemented in an in-house Fortran code for 2D axi-symmetric conditions (see Section 3), but the framework may readily be extended to 3D modelling, with the main challenge being related to the adopted image analysis technique for the determination

of "potentially active" plastic zones. The present framework builds on classical techniques such as connected component labelling and raster imaging, that can readily be extended from $2 \mathrm{D}$ pixels to $3 \mathrm{D}$ voxels.

It is demonstrated, throughout Section 4, how a robust numerical approach to handling the generalized flow theory gives close correspondence with visco-plastic analyses. The three benchmark tests considered clearly show that the new model readily handles repeated loading/unloading and the interaction of multiple plastic zones at large rigid body rotations/motions combined with finite strain deformation.

\section{Acknowledgements}

The work is financially supported by the VILLUM FOUNDATION Young Investigator Programme in the project "A New phenomenon Yet to be resolved in ductile PLATE tearing", grant VKR023451, and by the Danish Council for Independent Research in the projects "Advanced Damage Models with InTrinsic Size Effects", grant DFF-701700121. 


\section{Appendix A. The effect of the volume change rate}

The round notched tensile bar is considered in the following accounting for either; i) the full non-symmetric system matrix including the volume change rate, and ii) an approximate symmetric system matrix omitting the volume change rate (that originates from elastic deformation). The boundary value problem is that of the second benchmark test (see Section 4.2), and the calculation is conducted for two distinct length parameters and low conventional strain hardening, $N=0.05$ (all other material properties are listed in Tab. A.1).

The load-deflection curves in Fig. A.1 clearly illustrate that the effect of neglegting the volume change rate is very small. The largest difference between two associated curves (being at an overall deformation of $\Delta / H=0.15$ ) is $0.6 \%$ for $L_{D} / W=0.02$ and slightly lower at $0.34 \%$ for $L_{D} / W=0.4$. Due to the very small effect of the volume change, its effect is omitted throughout the present work and the numerical solution methods are based on symmetric system matrices.

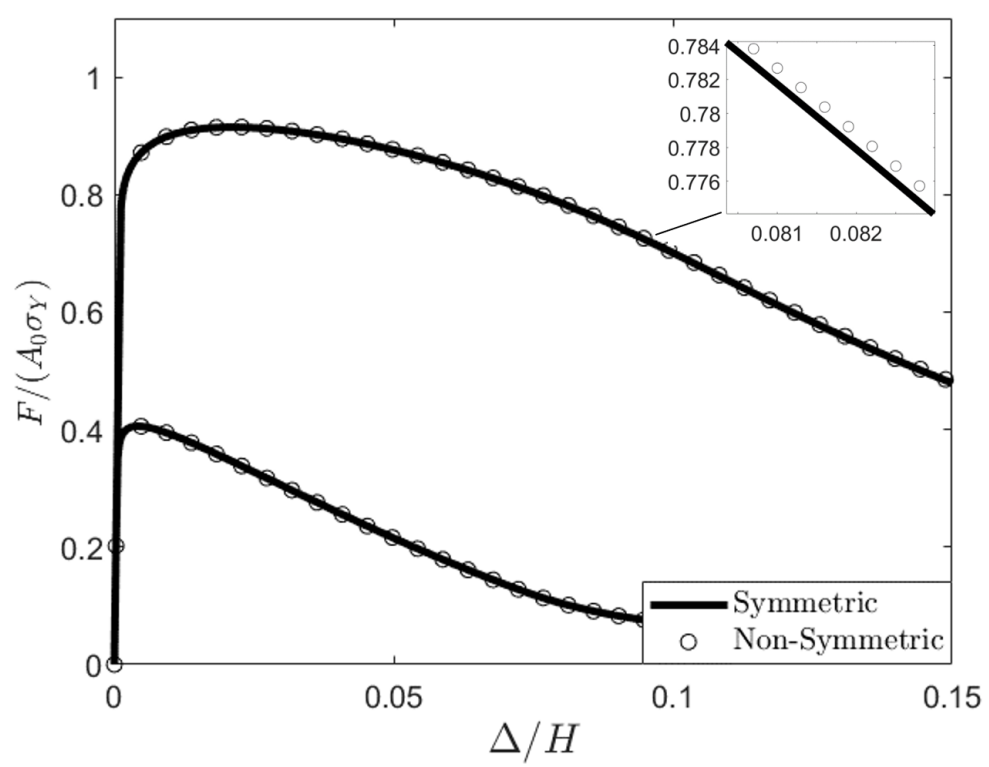

Figure A.1: Load-deflection curves for a round notched tensile bars for two different length parameters $\left(L_{D} / W=0.02\right.$ and $\left.L_{D} / W=0.4\right)$, demonstrating the negligible effect of the volume change rate.

\section{References}

Dahlberg, C., Faleskog, J., 2012. An improved strain gradient plasticity formulation with energetic interfaces: theory and a fully implicit finite element formulation. 
Comput. Mech.: DOI 10.1007/s00466-012-0743-5.

Danas, K., Deshpande, V., Fleck, N., 2012. Size effects in the conical indentation of an elasto-plastic solid. J. Mech. Phys. Solids. 60, 1605-1625.

Fleck, N., Hutchinson, J., 2001. A reformulation of strain gradient plasticity. J. Mech. Phys. Solids 49, 2245-2271.

Fleck, N., Willis, J., 2009a. A mathematical basis for strain-gradient plasticity theory. Part I: Scalar plastic multiplier. J. Mech. Phys. Solids 57, 161-177.

Fleck, N., Willis, J., 2009b. A mathematical basis for strain-gradient plasticity theory. Part II: Tensorial plastic multiplier. J. Mech. Phys. Solids 57, 1045-1057.

Fredriksson, P., Gudmundson, P., 2005. Size-dependent yield strength of thin films. Int. J. Plast. 21, 1834-1854.

Gudmundson, P., 2004. A unified treatment of strain gradient plasticity. J. Mech. Phys. Solids 52, 1379-1506.

Gurtin, M., Anand, L., 2005. A theory of strain-gradient plasticity for isotropic, plastically irrotational materials. Part I: Small deformations. J. Mech. Phys. Solids 53, 1624-1649.

Haralick, R., Shapiro, L., 1992. Computer and Robot Vision. Volume I, AddisonWesley, 28-48.

Martínez-Pañeda, E., Deshpande, V., Niordson, C., Fleck, N., 2019. Length scales in plasticity and fracture. (submitted for publication).

McMeeking, R., Rice, J., 1975. Finite-element formulations for problems of large elasticplastic deformation. Int. J. Solids Struct. 11, 601-616.

Nielsen, K., Niordson, C., 2012. Rate sensitivity of mixed mode interface toughness of dissimilar metallic materials. Int. J. Solids. Struct. 49, 576-583.

Nielsen, K., Niordson, C., 2013. A 2D finite element implementation of the Fleck-Willis strain-gradient flow theory. Europ. J. Mech. A/Solids 41, 134-142. 
Nielsen, K., Niordson, C., 2014. A numerical basis for strain-gradient plasticity theory: rate-independent and rate-dependent formulations. J. Mech. Phys. Solids 63, 113127.

Niordson, C., Hutchinson, J., 2011. Basic strain gradient plasticity theories with application to constrained film deformation. J. Mech. Mater. Struct. 6, 395-416.

Niordson, C., Legarth, B., 2010. Strain gradient effects on cyclic plasticity. J. Mech. Phys. Solids 58, 542-557.

Niordson, C., Redanz, P., 2004. Size-effects in plane strain sheet-necking. J. Mech. Phys. Solids 52, 2431-2454.

Niordson, C. F., Tvergaard, V., 2018. A homogenized model for size-effects in porous metals. J. Mech. Phys. Solids (in press).

Yamada, Y., Sasaki, M., 1995. Elastic-plastic large deformation analysis program and lamina compression test. Int. J. Mech. Sci. 37, 691-707.

\section{Tables}

Table A.1: Material properties.

\begin{tabular}{|c|c|c|}
\hline Parameter & Significance & Value \\
\hline$\sigma_{Y} / E$ & Initial yield strain & 0.001 \\
\hline$\nu$ & Poisson ratio & 0.3 \\
\hline$N$ & Strain hardening exponent & $0.05-0.2$ \\
\hline$m$ & Strain rate hardening exponent & 0.002 \\
\hline$\dot{\varepsilon}_{0}$ & Reference strain rate & $0.001^{*}$ \\
\hline$L_{D} / W$ & Normalized dissipative length parameter & {$[0.02,0.1,0.2,0.4]$} \\
\hline
\end{tabular}




\section{Figures}

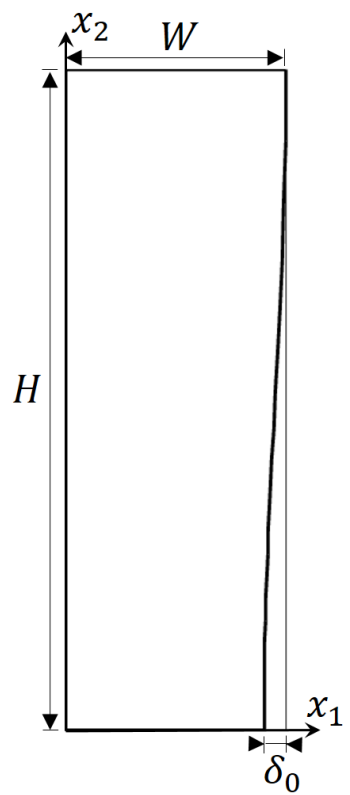

(a)

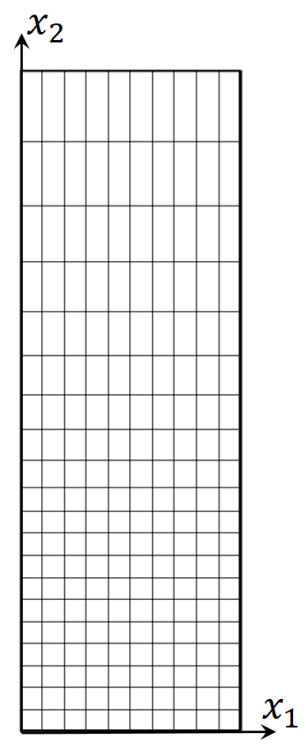

(b)

Figure 1: a) Schematic of the first benchmark test considering a round uni-axial tensile bar of length, $2 H$, and radius, $W$, with a smooth thinning introduced according to; $\delta\left(x_{2}\right)=-\delta_{0}\left(1+\cos \left(\pi x_{2} / H\right)\right) / 2$, where $\delta_{0}$ sets the imperfection size. b) A structured finite element mesh. Uni-axial stretching is prescribed at the top boundary (at $x_{2}=H$ ), while symmetry conditions are prescribed along $x_{2}=0$ and along the center axis (at $\left.x_{1}=0\right)$. 


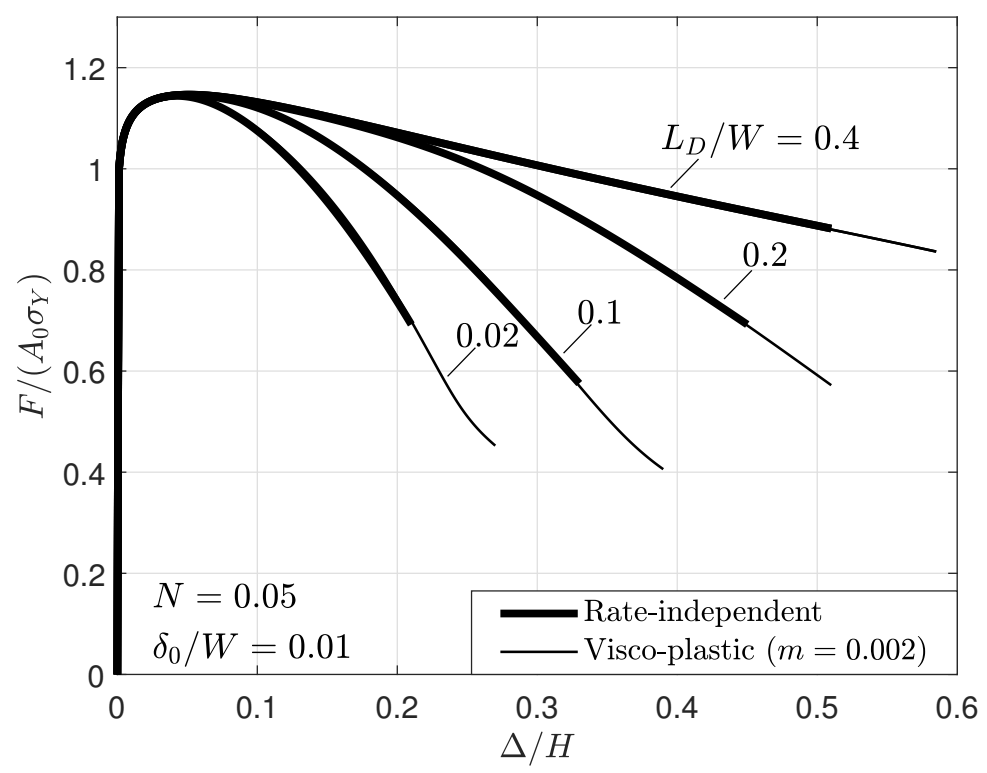

(a) Load-deflection curves
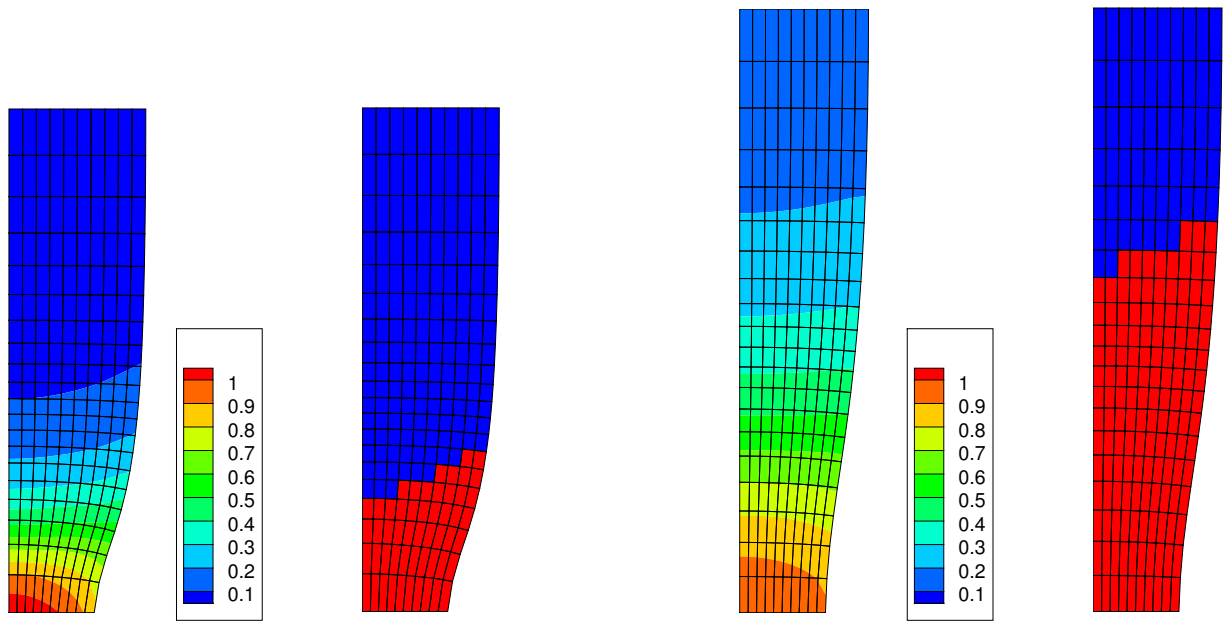

(b) Contours of $E^{p}$ (left) and active plastic zone (c) Contours of $E^{p}$ (left) and active plastic zone (right) for $L_{D} / W=0.02$ at $F /\left(A_{0} \sigma_{Y}\right) \approx 0.7$. (right) for $L_{D} / W=0.2$ at $F /\left(A_{0} \sigma_{Y}\right) \approx 0.7$.

Figure 2: a) Load-deflection curves for round uni-axial tensile bars for four different length parameters, and corresponding deformed configurations after peak load is attained and the load has dropped to; $F /\left(A_{0} \sigma_{Y}\right) \approx 0.7$. Here, showing the contours of the gradient enhanced effective plastic strain, $E^{p}$, and the active plastic zone for b) $L_{D} / W=0.02$ and c) $L_{D} / W=0.2$. 


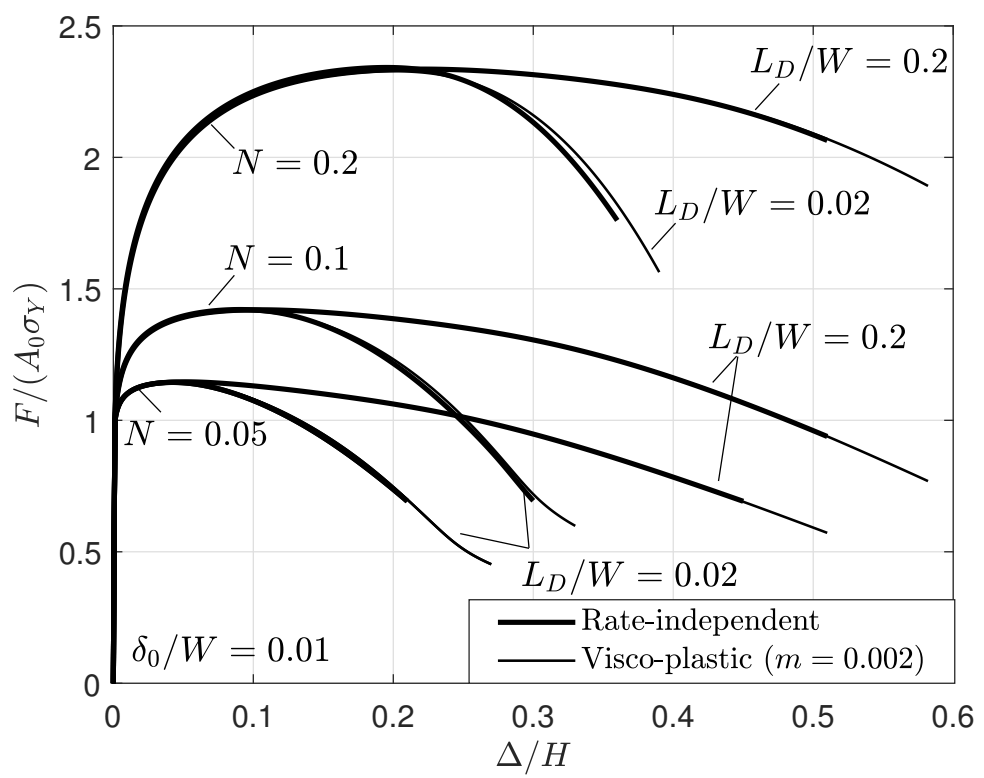

(a)

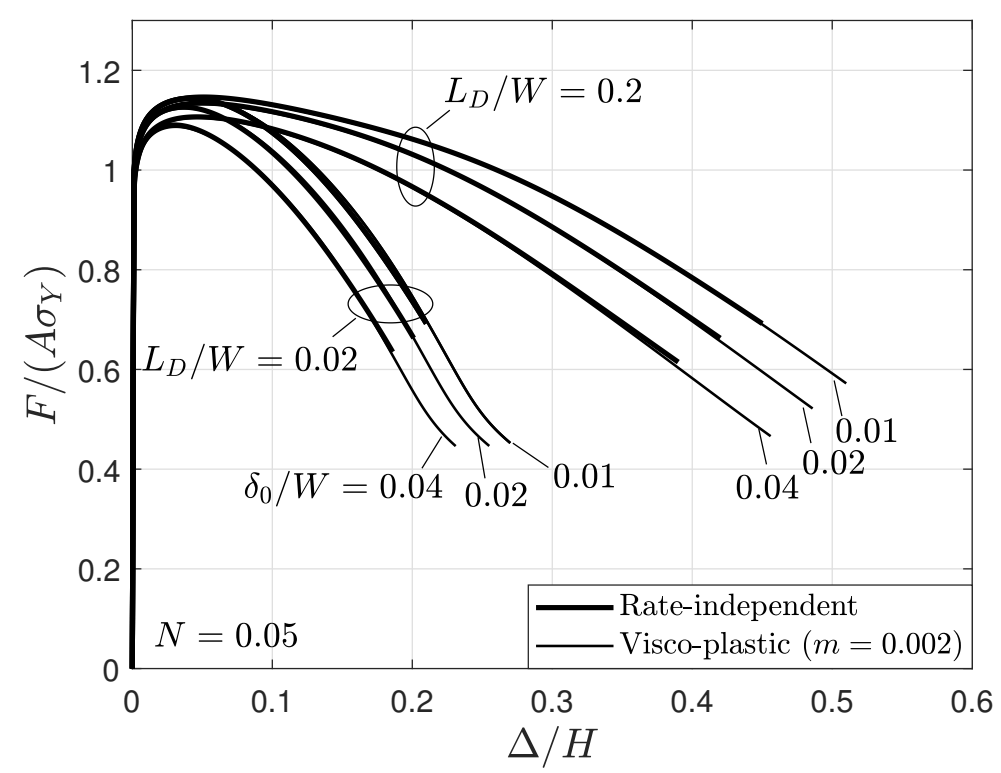

(b)

Figure 3: Load-deflection curves for round uni-axial tensile bars for various parameter sets. a) showing the effect of the length parameter for three values of strain hardening, and b) demonstrating the effect of the imposed imperfection on the radius. 


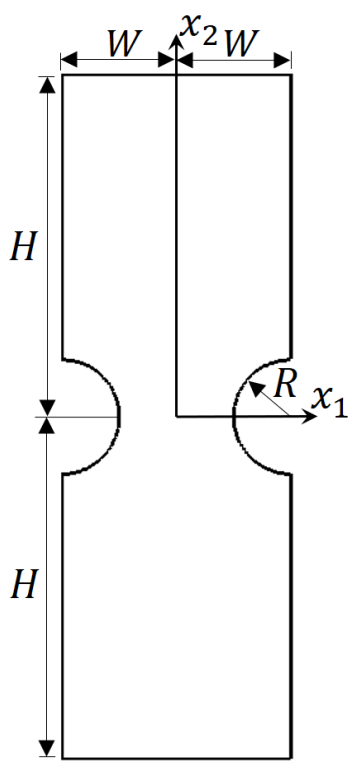

(a)

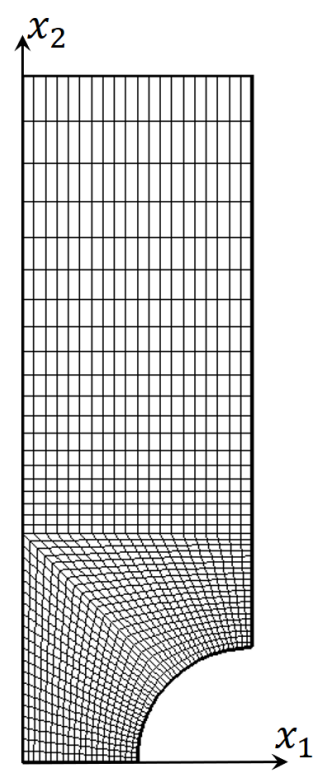

(b)

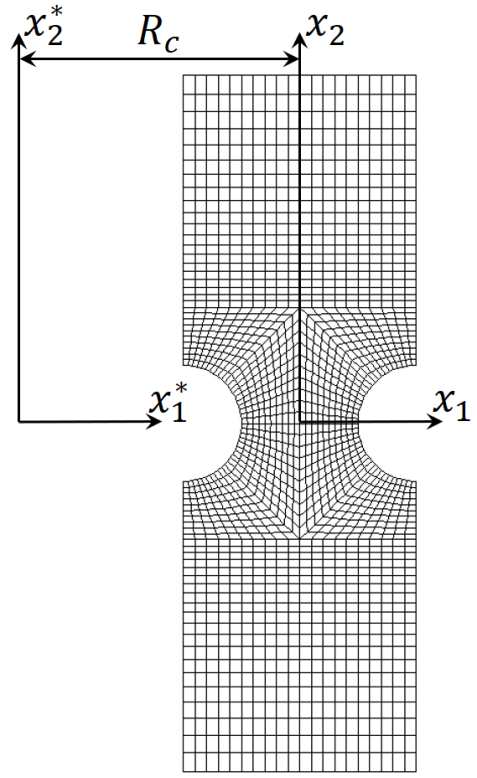

(c)

Figure 4: a) Schematic of the second and third benchmark tests considering a domain of length, $2 H$, radius (or width for test three), $W$, and notch size, $R$. The domain is, throughout, characterized by having $H / W=3$ and $R / W=0.5$. b) The discretized domain for the second benchmark test, and c) The discretized domain for the third benchmark test. 


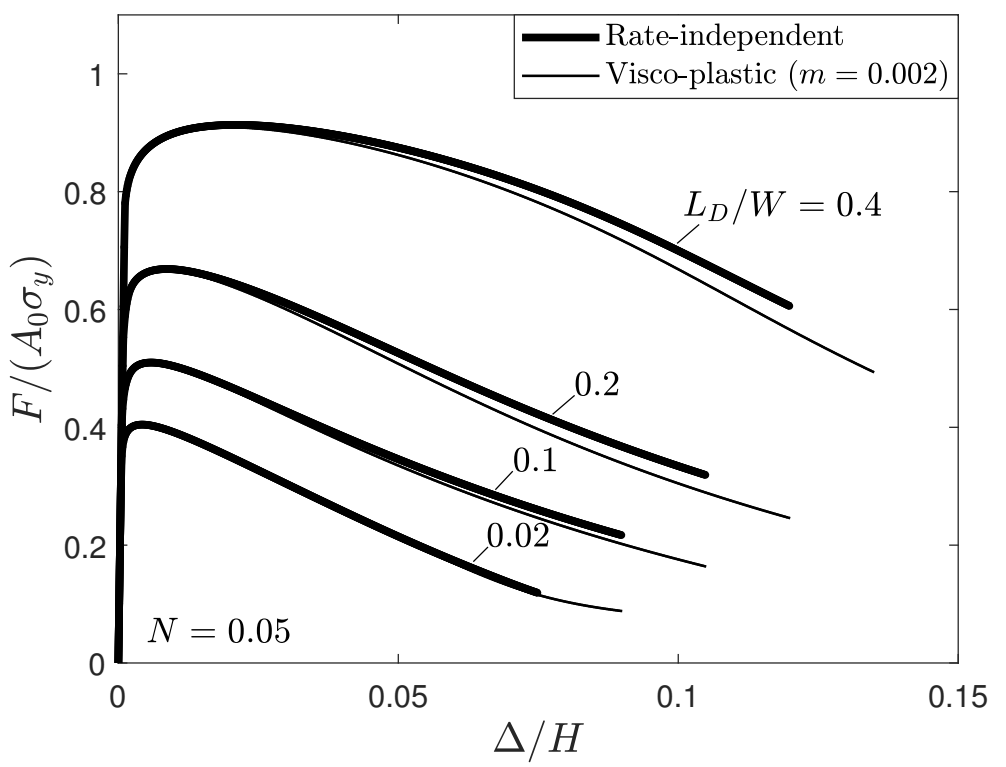

(a)

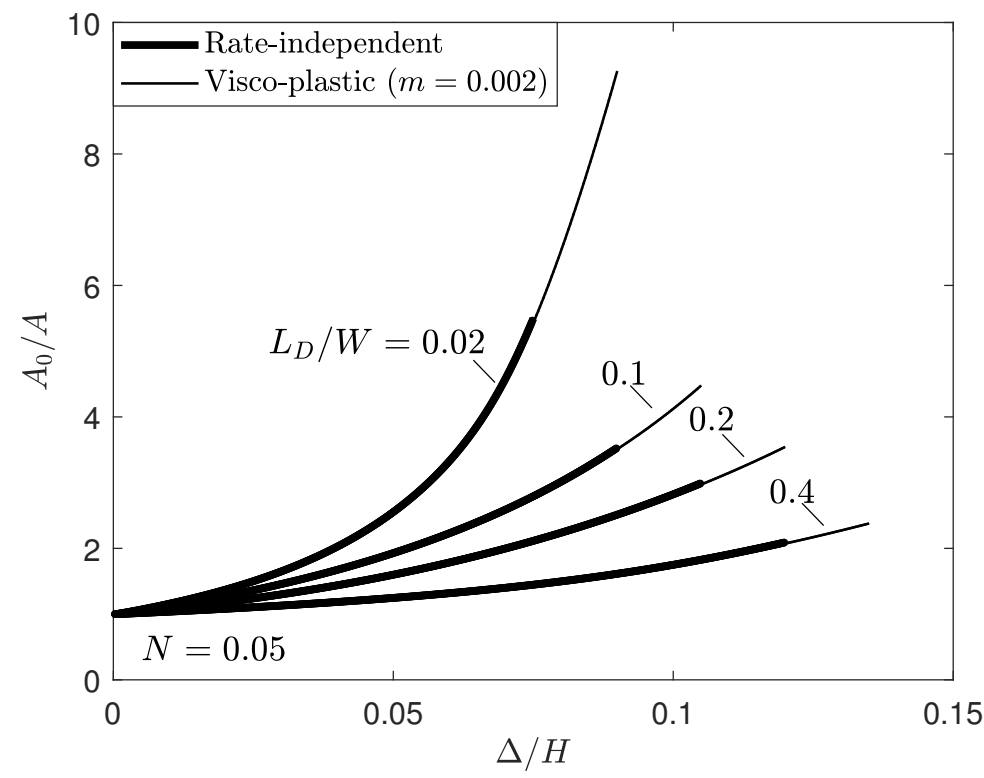

(b)

Figure 5: Predicted response for notched round tensile bars for increasing length parameter, showing a) the load deflection curves for low conventional strain hardening $(N=0.05)$, and b) the relative thinning of the cross-section of the notch (at $x_{2}=0$, see Fig. $4 \mathrm{~b}$ ). 

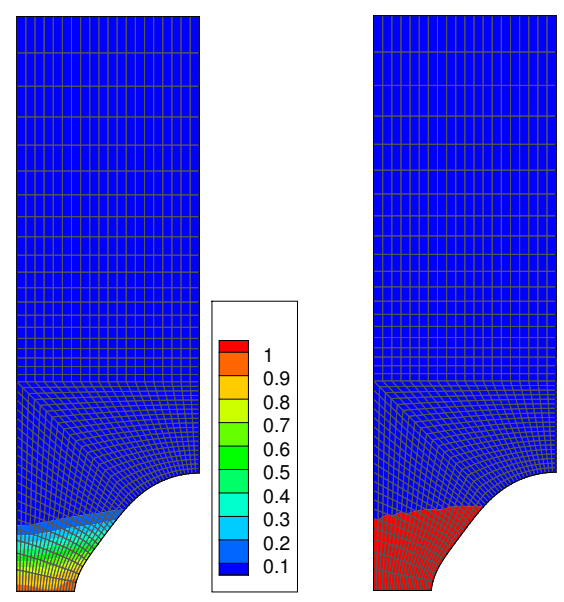

(a) Contours of $E^{p}$ (left) and active plastic zone (right) for $L_{D} / W=0.02$ at $\Delta / H=0.05$.
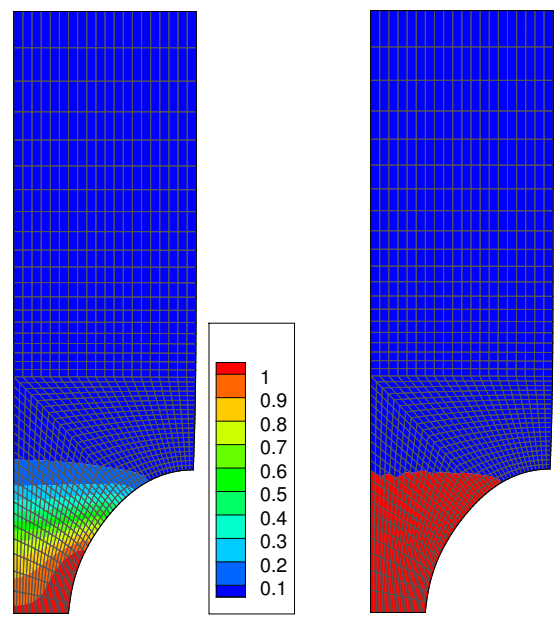

(b) Contours of $E^{p}$ (left) and active plastic zone (right) for $L_{D} / W=0.2$ at $\Delta / H=0.10$.

Figure 6: Deformed configurations of the notched bar, showing the contours of the gradient enhanced effective plastic strain, $E^{p}$, and the active plastic zone for a) $L_{D} / W=0.02$ at $\Delta / H=0.05$ and b) $L_{D} / W=0.2$ at $\Delta / H=0.10$.

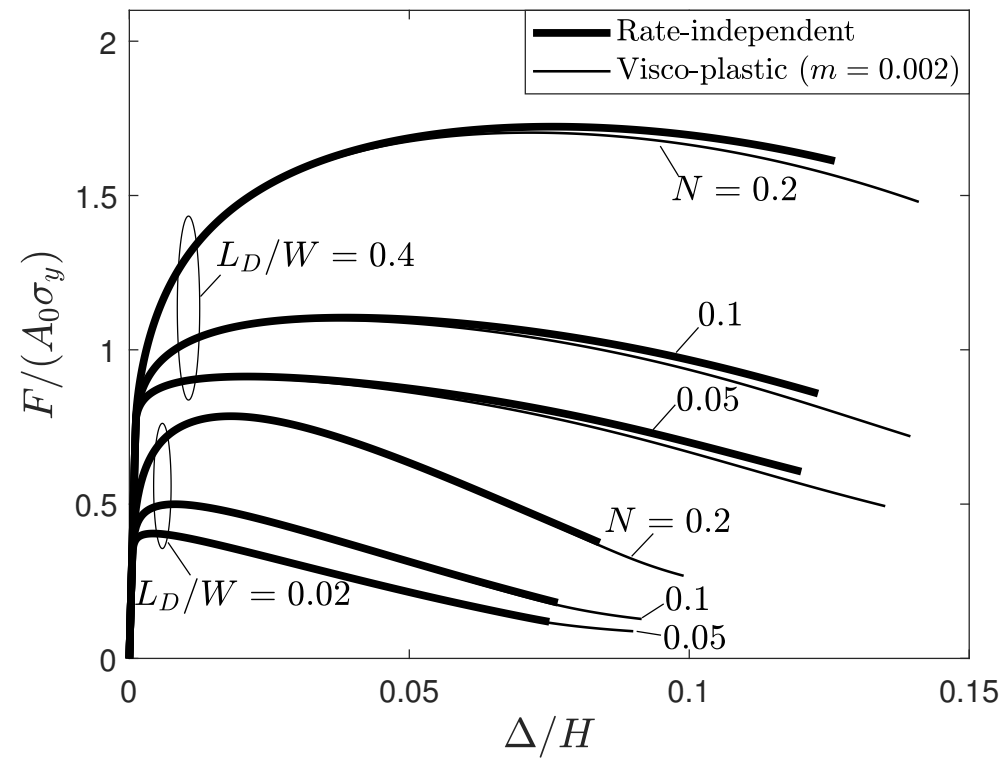

Figure 7: Load-deflection curves for notched round tensile bars for two distinct length parameters $\left(L_{D} / W=0.02\right.$ and $\left.L_{D} / W=0.4\right)$ at three levels of conventional strain hardening $(N=[0.05,0.1,0.2])$. 


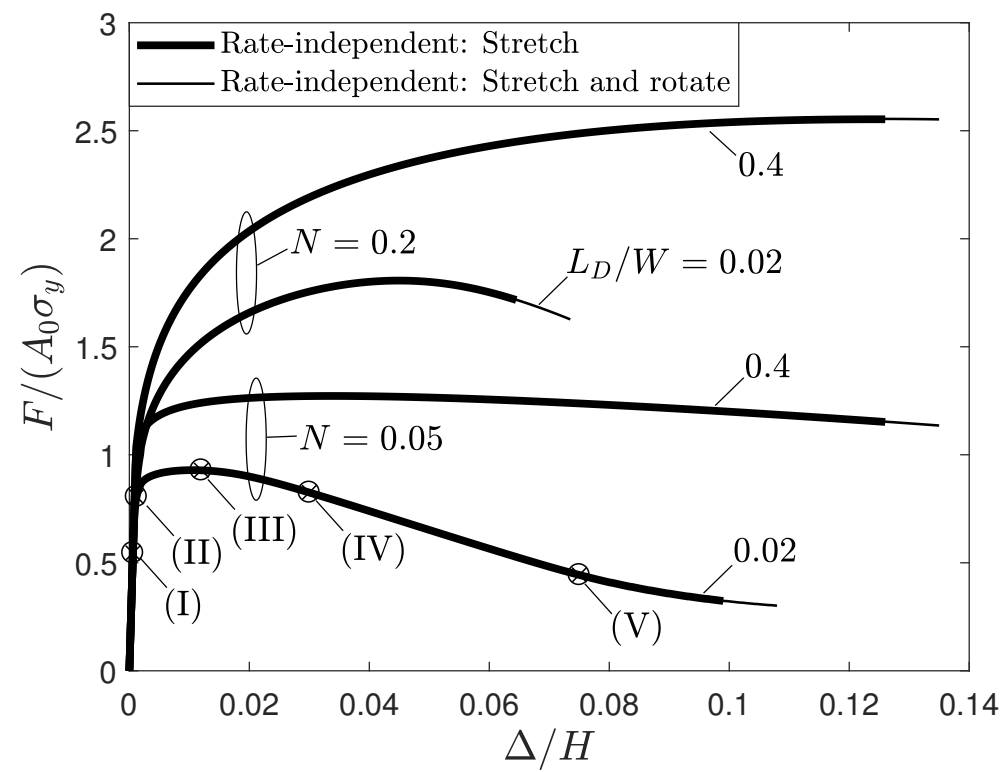

Figure 8: Load-deflection curves of notched large rings subject to tension (the third benchmark test) with $N=[0.05,0.2]$ and $L_{D} / W=[0.02,0.4]$. Here, showing two distinct loading scenarios; i) the first being simple stretching, and ii) the second being combined stretching and rotation of the domain. 


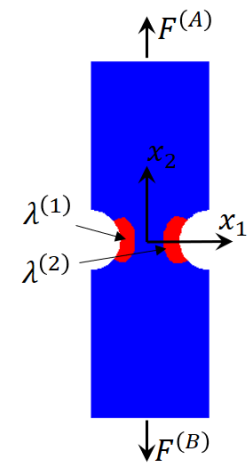

(I)

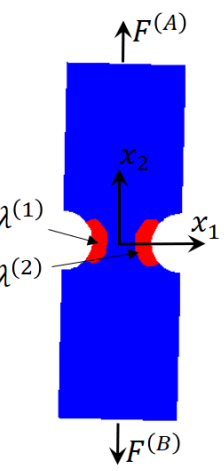

(I)

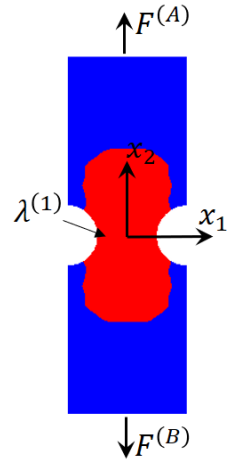

(II)

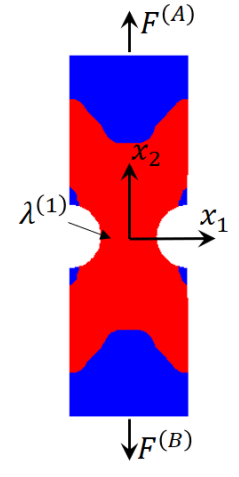

(III)

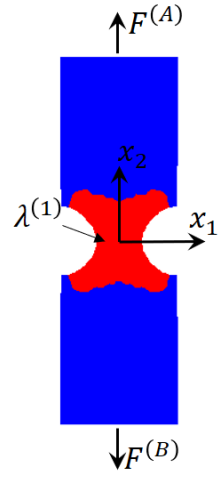

(IV)

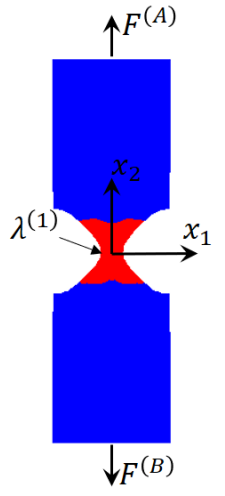

(V)

(a) Stretch

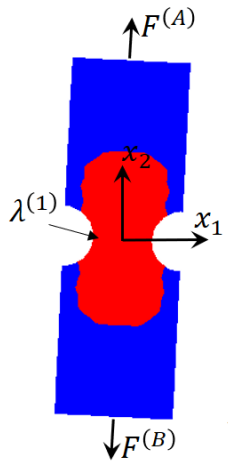

(II)

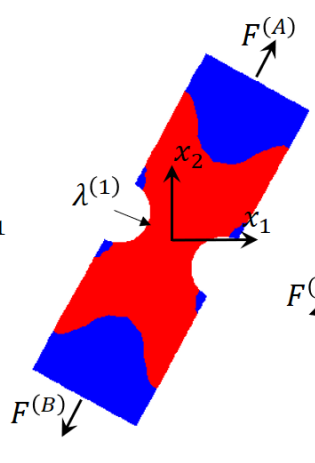

(III)

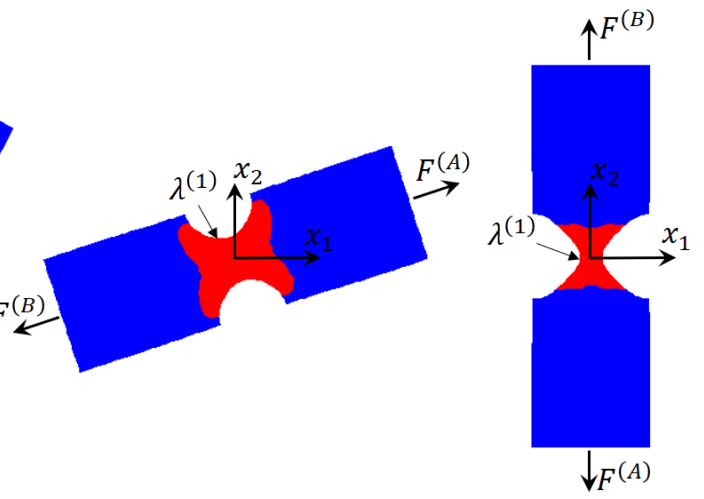

(IV)

(b) Stretch, $\Delta$, and rotate, $\theta$, fixing the ratio at $\theta /(\dot{\Delta} / H)=4 \pi / 3$.

Figure 9: Deformed configuration of the notched large ring with $L_{D} / W=0.02$ and for the two distinct loading scenarios. Here, showing the evolution of the active plastic zone marked their plastic multipliers for a) when stretching the domain, and b) when stretching is combined to a finite rigid body rotation. The deformation states correspond to those encircled on Fig. 8. 